\title{
Hinweise auf Vorteile bei Therapie mit Insulinanalogon
}

\begin{abstract}
Mit Insulinaspart kommt es zu weniger kardiovaskulären Ereignissen als mit Humaninsulin. Zu diesem Ergebnis kam eine retrospektive Analyse der Daten von mehr als 6000 Typ-2-Diabetikern, die in deutschen Allgemeinarztpraxen behandelt wurden.
\end{abstract}

Für die Untersuchung wurde eine Datenbank herangezogen, in der die Patientendaten, Diagnosen und Arzneiverordnungen aus 1919 Allgemeinarztpraxen registriert waren. Die Hälfte der Patienten hatte Insulinaspart bekommen, die andere Hälfte Humaninsulin (jeweils $3154 \mathrm{~Pa}$ tienten im Alter von durchschnittlich 60 Jahren).

Nach 3,5 Jahren war bei 27,4\% der Patienten mit dem Insulinanalogon und bei 30,6\% der mit Humaninsulin behandelten Patienten ein makrovaskuläres Ereignis (koronare Herzkrankheit, Herzinfarkt, Schlaganfall, TIA oder PAVK) aufgetreten. Dies entspricht einer relativen Risikoreduktion von 15\%. Im Einzelnen war die Häufigkeit von Schlaganfällen um $42 \%$, von Herzinfarkten um $31 \%$ und einer PAVK um 20\% geringer, wenn das
Insulinanalogon verordnet wurde. Bei mikrovaskulären Komplikationen zeigten sich hingegen keine Unterschiede. Der $\mathrm{HbA}_{1 \mathrm{c}}$-Wert war mit dem Insulinanalogon etwas niedriger als mit Humaninsulin (7,9 vs. $8,3 \%$; $\mathrm{p}<0,05)$.

Aufgrund des retrospektiven Studiendesigns lässt sich noch nicht endgültig der Rückschluss ziehen, dass Insulinaspart besser vor kardiovaskulären Ereignissen schützt. Dennoch vermuten die Autoren, dass die unterschiedliche Pharmakokinetik der Insuline nicht nur zu besseren postprandialen Blutzuckerwerten mit dem Insulinanalogon beiträgt, sondern auch positive Effekt auf die Gefäße haben könnte.

Auch in der NICE-(Nippon Ultra-Rapid Insulin and Diabetic Complications Evaluation-)Studie, einer kleinen, rando-

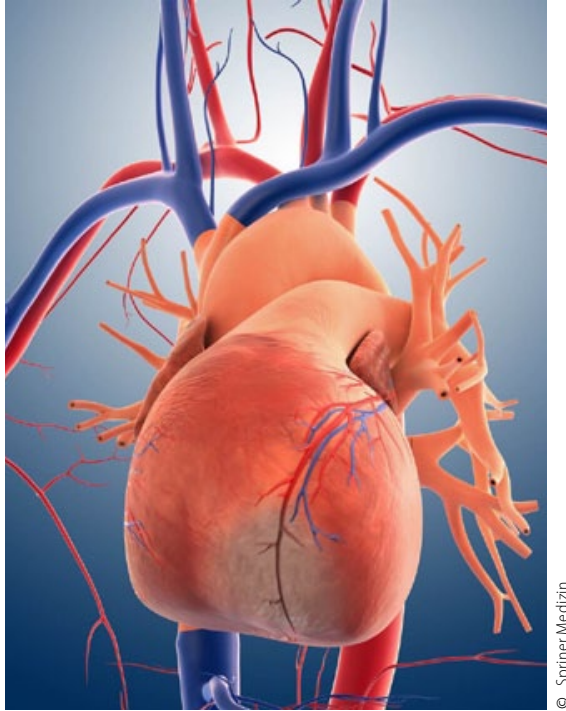

Kann die Wahl des Insulins das Herzinfarktrisiko beeinflussen?

misierten, kontrollierten, über fünf Jahre laufenden Studie mit 325 Typ-2-Diabetikern aus Japan, waren mit dem Insulinanalogon weniger kardiovaskuläre Ereignisse aufgetreten als mit Humaninsulin. Die Reduktion betrug hier $43 \%$.

Dr. Judith Neumaier

Quelle: Rathmann W, Kostev K. Diabetes Obes Metab. 2013 Apr;15(4):358-63. doi: 10.1111/ dom.12035. Epub 2012 Nov 28.

\section{Bei der Arzneiwahl zählen auch Risiko für Gewichtszunahme und Hypoglykämien}

\author{
Bei Typ-2-Diabetes kommt es nicht nur auf die Blutzuckerwerte an, son- \\ dern auf das kardiovaskuläre Gesamtrisiko. Hier sind auch Körpergewicht \\ und Hypoglykämierisiko wichtig. Inkretinbasierte Antidiabetika wie DPP- \\ 4-Hemmer sind in dieser Hinsicht von Vorteil, weil sie beides nicht erhöhen.
}

„Den Zucker korrigieren kann jeder, aber
ich möchte Ihr Problem ganzheitlich an-
gehen“, mit diesen Worten beginnt Dr.
Rainer Betzholz, niedergelassener Diabe-
tologe in Neuss, meist seine Erstgesprä-
che mit Typ-2-Diabetikern. Es gehe nicht
nur um die Glukosewerte, sondern um
das kardiovaskuläre Gesamtrisiko. Hier-
auf haben auch das Gewicht und das Hy-
poglykämierisiko einen Einfluss. Denn
schwere Hypoglykämien erhöhen die
Rate makrovaskulärer Ereignisse um den
Faktor 2,9 und die kardiovaskuläre Sterberate um den Faktor 2,7 (Zoungas S, et al. N Engl J Med. 2010;363:1410-8). Laut Prof. Werner Kern vom Zentrum für Hormon- und Stoffwechselerkrankungen in Ulm führen nächtliche Hypoglykämien, die oft unbemerkt bleiben, zu erhöhter Nahrungsaufnahme am nächsten Tag und beeinträchtigen das Gedächtnis.

Betzholz strebt bei Typ-2-Diabetern individuelle Therapieziele an und versucht zunächst meist, auf Arzneien zu verzich- ten, um die Motivation für die Lebensstiländerung nicht zu beeinträchtigen. Liegt eine atherothrombotische Gefäßerkrankung vor, verordnet er jedoch Metformin, wie von der DDG empfohlen. Der Grund: Das Sterberisiko war den Daten des REACH-Registers zufolge mit Metformin reduziert (Roussel $\mathrm{R}$ et al. Arch Intern Med. 2010;170(21):1892-9). Bei unzureichendem Ansprechen bevorzugt Betzholz als weiteres orales Antidiabetikum eine inkretinbasierte Therapie, etwa mit einem DDP-4-Hemmer, da diese weder das Gewicht noch das Hypoglykämierisiko erhöht (Phung OJ, et al. JAMA 2010;303: 1410-8). Kardiovaskuläre Endpunktstudien mit DPP-4-Hemmern, die kardiovaskulären Vorteil belegen sollen, laufen. Erste Ergebnisse erwartet Betzholz 2014 für Sitagliptin (z.B. Xelevia ${ }^{\varpi}$ ). Petra Eiden

Quelle: Symposium „Typ-2-Diabetes 2012: Lebensstil im Blick - die Therapie "im Genick", 16.11.2012, DDG-Herbsttagung in Berlin, Veranstalter: Berlin-Chemie 